\title{
Determining Motoric Abilities of Perspective Judokas
}

\author{
Dragan Popovic, Milos Popovic, Jasna Popovic, Evagelia Boli, Vladimir Savic,Ivana Arsic and Sonja Antonijevic \\ Faculty of Sport and Physical Education, University of Pristina, Leposavic 38218, Serbia
}

\begin{abstract}
The research was conducted on a sample of 237 perspective judokas with the objective of checking the efficiency of the factor analysis as an optimal method of establishing of the real structure of motor abilities. In order to assess motor abilities 20 motor tests have been applied, which have been selected so that analysis could be done on the basis of second order factors. All the data in this study were processed at the Multidisciplinary Research Center, Faculty of Sport and Physical Education, University of Pristina through the system of data processing software programs. Factor structure analysis of motor dimensions indicates that three factors were obtained: the first factor is responsible for movement structuring, the second factor is for the excitation intensity regulation and the third is responsible for the excitation duration.
\end{abstract}

Key words: Factor analysis, judokas, method, structure, motor abilities, reliability.

\section{Introduction}

The structure of motoric abilities has been analyzed a number of times, at different age, with different system of variables and by means of different methodological procedures. Most of the research was based on classical phenomenological, and some of them on functional method.

Lately, a hypothetical model of hierarchical motoric dimension structure is a current model with us, which has been defined and suggested by [1].

The assumed model of motoric dimension structure was checked by [1] by applying 110 motoric tests, on the sample of 693 male respondents, aged from 19 to 27 years old.

The results of that research have shown that the structure of motoric dimensions is organized hierarchically on three levels, but that the obtained structure shows significant deviation from the hypothetical model. The structure of the obtained model was more complex, so functional, rather than structural analysis had to be applied with certain models. The aforementioned model of hierarchical structure of motoric abilities and the results of its

Corresponding author: Dragan Popovic, Ph.D., professor, research fields: computation statistics, computer programming, mathematical psychology, kinesiology and motor control. testing by the same authors served as a starting point of this research.

Thus, there was an attempt in this study to do structure analysis at the level of second order factors, the model of which is given, and with the help of a factor of the first order, that is, with representative measuring instruments for the assessment of the primary factors. Hence, the area of motoric skills was covered with twenty tests.

\section{Methods}

An intercorrelation matrix of 20 measuring instruments for the assessment of motoric abilities was analyzed by the method of main components on the sample of 237 respondents. The sequence of variables and their symbols are the same as in the earlier research [5] and [7]. First of all, the intercorrelation matrix of those variables is factorized in order to determine the structure of motor variables. Typical values of intercorrelation matrix were calculated by algorithm modification which was proposed by householder, Ortega and Wilkinson.

The characteristic roots of matrix $\mathbf{R}$ are given in the table of their own values and marked with LAMBDA. Relative cumulative contribution of those characteristic roots is calculated and given in the row 
marked as CUMULATIVE. The characteristic roots can be interpreted as latent variable variances, defined as main components. For assessing the significance of the main components PB criterion is used which gives a minimal number of significant main components.

The last significant characteristic root, in accordance with the applied criterion, is marked as the last used own value. The size of the relative cumulative contribution with the characteristic root multiplied by 100 gives the percentage of variance of the entire system explained by predictor variables [2] and [3].

Significant characteristic vectors are denominated in such a way for their norm to be equal with corresponding characteristic roots. Thus obtained main components of intercorellation matrix of motoric variables are presented in Table 3. The coefficients in the table 2. mentioned above are the coordinates of the vector of variables on orthogonal system of latent dimensions defined as main components. Given the nature of motoric variables, the first main component can be seen as the first and the most important common object of measurement of all the applied measuring instruments.

In order to obtain a simple structure of isolated latent dimensions, without the restrictions required by the orthogonality of these dimensions, significant components are transformed in the oblimin position by direct application of oblimin methods by [2]. The applied oblimin criterion was calculated by minimizing the sum of independent vector products of variable square coordinates.

Communalities of motoric variables were calculated as scalar products of the parallel projection vector and the orthogonal projection vector of each variable on the factors in oblimin position. Those communalities were presented in column marked as $\mathrm{h} 2$. These values can be understood as a part of each motoric variable variance, which can be explained with an isolated system of latent dimensions. Communalities are concurrently the vector lengths of the manifest variables projected into space of isolated latent variables. The size of communalities is proportional to the size of the common variance which each variable has with a set of other, if the size of the variance depends on the common latent dimensions of the whole system of motoric variables [2]).

Coordinates, that is, parallel projections of the manifest variable vectors applied on the system defined by vectors of latent dimensions in oblimin position, are given in Table 3.

The relations between the isolated latent dimensions, that is, the correlations between factors in oblimin position, are shown in Table 4.

The correlations between manifest and latent variables, defined as oblimin factors are given in Table 5 .

\section{Discussion}

Judging by the intercorrelation matrix structure, the features of manifest variables which are applied in this research are not at a satisfactory level.

Although the intercorrelation matrix is not the best and the only indicator of basic features of a system of measuring instruments, it is still an irreplaceable source of information on the features of the basic space features for input condensation. According to this criterion a system of 20 motor variables is structured so as to form a couple of blocks, some of which have decent intercorrelations, some have satisfactory high intercorrelations, whereas some build basically only logical blocks, but have quite weak intercorrelations even within their own block (Table 1).

Three latent dimensions with a total of $52 \%$ of common variance are isolated on the basis of $\mathrm{PB}$ criterion.

The first main component with $33 \%$ of variance, the proportion it depletes from the total variability of the entire system of variables, acts as a general factor of motoric skills. All variables have high and most frequently maximum correlation with this factor which is also, without a doubt, the first main subject 
Table 1 Intercorrelations of motoric variables.

\begin{tabular}{|c|c|c|c|c|c|c|c|c|c|c|c|c|c|c|c|c|c|c|c|c|}
\hline & 1. & 2. & 3. & 4. & 5. & 6. & 7. & 8. & 9. & 10. & 11. & 12. & 13. & 14. & 15. & 16. & 17. & 18. & 19. & 20. \\
\hline 1. & 1.00 & & & & & & & & & & & & & & & & & & & \\
\hline 2. & .13 & 1.00 & & & & & & & & & & & & & & & & & & \\
\hline 3. & -.17 & -.25 & 1.00 & & & & & & & & & & & & & & & & & \\
\hline 4. & -.38 & -.32 & .47 & 1.00 & & & & & & & & & & & & & & & & \\
\hline 5. & .60 & .33 & -.35 & -.53 & 1.00 & & & & & & & & & & & & & & & \\
\hline 6. & -.37 & -.33 & .15 & .30 & -.17 & 1.00 & & & & & & & & & & & & & & \\
\hline 7. & -.42 & -.30 & .18 & .53 & -.61 & .44 & 1.00 & & & & & & & & & & & & & \\
\hline 8. & -.39 & -.29 & .21 & .45 & -.45 & -.41 & .55 & 1.00 & & & & & & & & & & & & \\
\hline 9. & .38 & .16 & -.09 & -.18 & .41 & -.52 & -.23 & -.30 & 1.00 & & & & & & & & & & & \\
\hline 11. & -.40 & -.44 & .26 & .55 & -.59 & .49 & .45 & .39 & -.26 & .63 & 1.00 & & & & & & & & & \\
\hline 12. & .20 & .21 & -.13 & -.27 & .45 & -.28 & -.42 & -.32 & .17 & -.27 & -.38 & 1.00 & & & & & & & & \\
\hline 13. & .08 & -.16 & .11 & .18 & -.08 & .08 & .04 & -.04 & .12 & .41 & .44 & -.18 & 1.00 & & & & & & & \\
\hline 14. & -.28 & -.26 & -.08 & .30 & -.42 & .23 & .34 & .21 & -.18 & .09 & .38 & -.24 & .07 & 1.00 & & & & & & \\
\hline 15. & -.27 & -.18 & -.03 & .06 & -.29 & .34 & .16 & .08 & -.22 & .12 & .35 & .16 & .10 & .34 & 1.00 & & & & & \\
\hline 16. & -.36 & -.36 & .28 & .49 & -.57 & .27 & .51 & .32 & -.18 & .48 & .56 & -.18 & .17 & .29 & .35 & .1.00 & & & & \\
\hline 17. & -.42 & -.35 & .28 & .44 & -.65 & .37 & .40 & .32 & -.39 & .44 & .46 & -.17 & .00 & .36 & .25 & .55 & 1.00 & & & \\
\hline 18. & -.08 & .06 & .05 & -.10 & -.26 & -.03 & .18 & .10 & -.02 & . 12 & .00 & -.20 & -.13 & .09 & .00 & .22 & .26 & . 1.00 & & \\
\hline 19. & -.09 & -.27 & .26 & .25 & -.24 & .23 & .23 & .11 & .02 & .48 & .49 & -.27 & .62 & .19 & .12 & .38 & .19 & .05 & 1.00 & \\
\hline
\end{tabular}


Table 2 Major axis of intercorrelation matrix of motoric variables.

\begin{tabular}{|c|c|c|c|c|c|}
\hline & FAC1 & FAC2 & FAC3 & $h^{2}$ & $\mathrm{U}$ \\
\hline MKOOP & -.58 & .37 & -.01 & .48 & .55 \\
\hline MS3ME & -.53 & .10 & .01 & .30 & .68 \\
\hline MTAPR & .41 & .10 & -.29 & .26 & .66 \\
\hline MTAPN & .68 & .00 & -.20 & .51 & .38 \\
\hline MPOLN & -.82 & .24 & .10 & .74 & .26 \\
\hline MDPNK & .62 & -.13 & .26 & .48 & .48 \\
\hline MPSNK & .70 & -.18 & -.23 & .58 & .41 \\
\hline MSUKL & .59 & -.26 & -.24 & .48 & .54 \\
\hline MISKR & -.44 & .40 & -.23 & .41 & .56 \\
\hline MSPAG & .66 & .28 & .03 & .52 & .38 \\
\hline MSUSM & .32 & .23 & .13 & .75 & .29 \\
\hline M20VS & -.49 & -.08 & .45 & .49 & .54 \\
\hline MBMIL & .30 & .79 & .14 & .75 & .42 \\
\hline MSKLR & .48 & -.17 & .15 & .30 & .63 \\
\hline MDNLE & .35 & -.14 & .75 & .71 & .53 \\
\hline MMSTT & .71 & -.03 &, 04 & .50 & .40 \\
\hline MINNS & .68 & -.28 & .64 & .55 & .41 \\
\hline MITUP & .15 & -.22 & -.37 & .21 & .65 \\
\hline MDSAK & .51 & .65 & .02 & .68 & .42 \\
\hline MFLIP & .37 & .72 & -.04 & .67 & .46 \\
\hline LAMBDA & 6.59 & 2.43 & 1.44 & & \\
\hline$\%$ & $33.00 \%$ & $12.00 \%$ & $7.00 \%$ & $51.63 \%$ & \\
\hline KUMUL. \% & $33.00 \%$ & $45.00 \%$ & & $52.00 \%$ & \\
\hline
\end{tabular}

Table 3 The composition of motoric factors.

\begin{tabular}{lrrr}
\hline & OBL1 & OBL2 & OBL3 \\
\hline MKOOP & .69 & .24 & -.10 \\
MS3ME & .35 & -.24 & -.15 \\
MTAPR & -.44 & .16 & -.28 \\
MTAPN & -.68 & .14 & -.14 \\
MPOLN & .88 & .07 & -.07 \\
MDPNK & -.49 & .03 & .34 \\
MPSNK & -.81 & -.05 & -.15 \\
MSUKL & -.76 & -.16 & -.16 \\
MISKR & .48 & .28 & -.38 \\
MSPAG & -.41 & .44 & .05 \\
MSUSM & -.51 & .45 & .21 \\
M20VS & .61 & -.14 & .47 \\
MBMIL & .20 & .91 & .06 \\
MSKLR & -.41 & -.04 & .27 \\
MDNLE & -.02 & .02 & .83 \\
MMSTT & -.60 & .14 & .12 \\
MINNS & -.71 & -.12 & .15 \\
MITUP & -.42 & -.23 & .34 \\
MDSAK & -.09 & .81 & .87 \\
MFLIP & .02 & -.01 \\
\hline
\end{tabular}


Table 4 Structure of motoric factors.

\begin{tabular}{lrrr}
\hline & OBL1 & OBL2 & OBL3 \\
\hline MKOOP & .64 & .00 & -.31 \\
MS3ME & .48 & -.37 & -.28 \\
MTAPR & -.41 & .28 & -.13 \\
MTAPN & -.68 & .35 & .07 \\
MPOLN & .85 & -.21 & -.26 \\
MDPNK & -.60 & .23 & .49 \\
MPSNK & -.75 & .19 & .08 \\
MSUKL & -.65 & .07 & .05 \\
MISKR & .49 & .09 & -.45 \\
MSPAG & -.58 & .59 & .22 \\
MSUSM & -.73 & .64 & .40 \\
M20VS & .51 & -.30 & .28 \\
MBMIL & -.11 & .84 & -.08 \\
MSKLR & -.98 & .11 & .39 \\
MDNLE & -.28 & .10 & .84 \\
MMSTT & -.69 & .35 & .31 \\
MINNS & -.71 & .11 & .35 \\
MITUP & -.24 & -.12 & .82 \\
MDSAK & -.35 & .81 & -.23 \\
MFLIP & -.21 & .08 \\
\hline
\end{tabular}

Table 5 Motoric factors correlations.

\begin{tabular}{lrrr}
\hline & OBQ1 & OBQ2 & OBQ3 \\
\hline OBQ1 & 1.00 & -.33 & -.31 \\
OBQ2 & -.33 & 1.00 & .09 \\
OBQ3 & -.31 & .09 & 1.00 \\
\hline
\end{tabular}

of the measurement of all elements of the motoric skills.

Therefore, the percentage of the remaining variability of $52 \%$ is successively divided to the remaining two components, of which $13 \%$ belongs to the second one, and only $7 \%$ to the third one. However, the second and the third main component still have informative value, even though they were formed on a considerably lower variance. Thus, certain amount of caution is necessary while interpreting them, especially if we take into consideration the main aim of this research.

The second main component is defined by the explosive strength assessment tests and by the maximum possible force rate development tests (in so-called attempted moves). The common feature of the first two tests is short-term muscle contraction caused by the maximum excitation of the central nervous system, which causes excitation of the maximum possible number of motor units. The feature of the other two tests which participate in defining a factor responsible for the excitation intensity regulation is the maximum possible force rate development under normal conditions.

Considering the fact that it depletes the variance the least, the third main component is not clearly defined. This dimension is in a way determined by three tests: first test for the repetitive strength assessment (MDNLE), the second test for the explosive strength assessment (M20VS) and the third test for the flexibility assessment (MDPNK).

As the test for repetitive strength assessment plays the greatest part in this component, it can be concluded with a certain amount of caution that the 
mechanism responsible for the duration of excitation basically lies in this factor.

Factorial structure of the motoric variables was analyzed concurrently, on the basis of all the information given orthoblique transformation of significant main components, that is, on the basis of variable correlation matrix and factors (Table 5) of parallel variable projections on factors (Table 2) and factor intercorrelation (Table 3).

The first factor is, according to the variance value, the most important one of the isolated dimensions. Its position in the coordinate system (obtained by orthoblique transformation) is general motoric factor. Apart from the tests for the force assessment (MFLLP, MDSAK), all the other tests have significant correlations with this dimension.

As it is not in a child's nature to solve motoric tasks in which high intensity energetic component prevails, and so-called "explosive strength" is more a characteristic of CNS which, because of significant genetic predetermination, can not be influenced, what remains is to analyze the problem of general motoric ability of pioneer judo athletes by means of information components of motoric tasks. This primarily refers to the structuring of movement (with different types of coordination), regulating the involvement of agonist and antagonist muscles and regulating muscle tone.

Functionally speaking, general motoric ability in children at this period of development probably depends on the effective function of the entire CNS. This efficacy is reflected in the quality of receptors, especially of kinesthetic, muscular, tendonal, afferent paths, decoder in a cortical and subcortical part of CNS, then in corticospinal and subcorticospinal paths, front motor neurons of a spinal cord, motoric units and the entire muscle effector [2] and [4].

The ability of manifesting greater physical strength at this age is not prominent. This primarily refers to generating muscle strength which is to move the body to another position. All contractions of isotonic and isometric character necessary for the realization of motoric tests applied in the program of motoric measurements provide information about the so-called "relative strength" and give the assessment of the corresponding muscle endurance. However, it is certain that such a partial motoric ability is not developed enough, which is a completely normal characteristic in ontogenetic development of man.

Since the speed of solving and performing of complex motor tasks is an essential feature of the content of most of the tasks, which still best define this dimension, it could be concluded that in the basis of this dimension lies the mechanism for structuring movements.

The second dimension is defined by: throwing a medicine ball from a lying position, left forearm flexion, hand dynamometer, standing long jump and a split. The common feature of the aforementioned tests, except the last one, is a short-term muscle contraction caused by the maximum excitation of the central nervous system, which causes the excitation of the maximum possible number of motor units. This type of muscle action results in a quick and explosive force development, and this dimension is most often defined as explosive strength. The projection of the variable of throwing a medicine ball from lying position also confirms the definition of the factor. The distance range is proportional to the square of the velocity of the throw (without considering other factors here).

The features of the tests - hand dynamometer and left forearm flexion are to develop maximum force possible under normal conditions (in so-called “attempted moves"). However, it must be emphasized that final input does not depend only on the current agonist activation, but, in fact, on the cumulative effect of successive involvement of a larger number of motor units so that the maximum number of muscle fibers is activated at the moment of maximum tension. During that, muscles are activated to generate force whose direction is not in accordance with the activated agonists, but serves to provide as much support as 
possible (that is, as fixators). Maximum muscle strength depends on the number of activated muscle units, and that number obviously is independent neither of the number of available units that can be activated at all nor, therefore, of the active muscle mass.

So the results in these tests do not only depend on the functional characteristics of the effector and on the excitability of the motor cortex, but also on the performance of subcortical centers that modulate the impulses from the motor cortex [5-7].

The third dimension is not clearly defined. Lifting legs lying down (MDNLE), $20 \mathrm{~m}$ running from a standing start (M20VS) and a flexibility assessment test (MDPNK) have the greatest projections on this dimension. The characteristic of the first test is that it requires long-term muscle strain; obviously in this case it is a discontinuous strain (repetition tests). The characteristics of the second test are the possibility of developing great excitation in primary motoric centers in the brain cortex and in those subcortical nuclei that function as amplifiers and efferent information modulators. In this case, this dimension has its explanation in the character of the movement regulation in primary motor centres and subcortical nuclei of CNS.

Since this dimension is highly correlated with the test MDNLE, it could be defined, with certain caution, as a latent functional structure that is responsible for regulating the duration of the excitation of the primary motor centers that control the movements of the active muscles [5-7].

\section{Conclusion}

It can be concluded from the obtained results that the factor analysis of the main components with oblimin transformation is a very suitable method for determining motor skills.

However, apart from the fact that at this moment this method is considered to be optimal exploration procedure, a number of further research is needed, which would continually complement the knowledge about the methods and laws of man's motoric skills.

\section{References}

[1] Gredelj, M., Metikos, D., Hosek, A., and Momirovic, K. 1975. "A Model of Hierarchical Structure of Motor Abilities." Kinesiology 5 (1-2): 7-81.

[2] Popovic, D. 1993. Programs and Subprograms for the Analysis of Quantitative Modifications. Monograph, Multidisciplinary Research Center, Faculty of Physical Education, Pristina, Serbia.

[3] Momirovic, K., and Popovic, D. 2003. Construction and Application of Taxonomic Neural Networks. Monography, Multidisciplinary Research Center, Faculty of Physical Education, Leposavic, Serbia

[4] Popovic, D., Boli, E., Popovic, M., Savic, V., Popovic, J., and Bojovic, M. 2016. "Determination of Differences in Motor Dimensions between Judo and Karate Athletes." Journal of Sports Science 4: 170-4.

[5] Popovic, D. 1990. Martial Arts I (Judo and Self-Defense). University of Nis. (in Serbian)

[6] Popovic, D. 1993. Determination of the Structure of Psychosomatic Dimensions in Combats and Development of Methods for Their Evaluation and Monitoring. Monography, University of Priština, Faculty of Physical Education, Pristina.

[7] Popović, D. 1990. "Metodologija istraživanja u fizičkoj kulturi. (Research Methodology in Physical Education)." University of Nis. (in Serbian) 